\title{
Electromagnetic Nucleon-to-Delta Transition in Chiral Effective-Field Theory
}

\author{
Vladimir Pascalutsa* and Marc Vanderhaeghen ${ }^{\dagger}$ \\ Physics Department, The College of William \& Mary, Williamsburg, Virginia 23187, USA \\ Theory Group, Jefferson Lab, 12000 Jefferson Avenue, Newport News, Virginia 23606, USA
}

(Received 5 August 2005; published 28 November 2005)

\begin{abstract}
We perform a relativistic chiral effective-field theory calculation of pion electroproduction off the nucleon $\left(e^{-} N \rightarrow e^{-} N \pi\right)$ in the $\Delta(1232)$-resonance region. After fixing the three low-energy constants, corresponding to the magnetic $(M 1)$, electric $(E 2)$, and Coulomb $(C 2) \gamma N \Delta$ couplings, our calculation provides a prediction for the momentum transfer and pion-mass dependence of the $\gamma N \Delta$ form factors. The prediction for the pion-mass dependence resolves the discrepancy between the recent lattice QCD results and the experimental value for the " $C 2 / M 1$ ratio" at low $Q^{2}$.
\end{abstract}

DOI: 10.1103/PhysRevLett.95.232001

PACS numbers: 12.39.Fe, 13.40.Gp, 13.60.Le

The $\Delta(1232)$ resonance, the first excited state of the nucleon, dominates many nuclear phenomena at energies between the one- and two-pion production thresholds. The electromagnetic excitation of the $\Delta$ resonance, the $\gamma N \Delta$ transition, has recently received a lot of attention. At low momentum transfer $\left(Q^{2}\right)$ it highlights the role of the pion cloud [1-7], whereas at larger $Q^{2}$ it probes the onset of the perturbative QCD regime [8,9].

The $\gamma N \Delta$ transition is predominantly of the magnetic dipole (M1) type which, in a simple quark-model picture, is described by a spin flip of a quark in the $s$-wave state. Any $d$-wave admixture in the nucleon or the $\Delta$ wave functions allows for the electric (E2) and Coulomb (C2) quadrupole transitions. Therefore, by measuring these, one is able to assess the presence of the $d$-wave components and hence quantify to which extent the nucleon or the $\Delta$ wave function deviates from the spherical shape ("hadron deformation") [10].

The $\gamma N \Delta$ transition has been accurately measured in the pion photo- and electroproduction reactions $[1-3,9]$. The $E 2$ and $C 2$ are found to be relatively small-the ratios $R_{E M}=E 2 / M 1$ and $R_{S M}=C 2 / M 1$ are at the level of a few percent. On the theoretical side, the most recent stateof-the-art lattice QCD study [11] obtained a puzzling result: the computed ratio $R_{S M}$ at low momentum transfer appears to be significantly different from the observed value. It is important to note that the lattice calculations were done at larger pion masses, while the result compared with experiment was obtained by a linear extrapolation to the physical pion mass.

In this Letter we present a first chiral effective-field theory $(\chi$ EFT) calculation of pion photo- and electroproduction on the nucleon in the $\Delta$-resonance region. Besides finding good agreement of our calculation with observables, we are able to study the chiral behavior $\left(m_{\pi}\right.$ dependence) of the $\gamma N \Delta$ transition. Our results show that there is no apparent discrepancy between the lattice data [11] and the experimental result for $R_{S M}$.

Our starting point is the relativistic chiral Lagrangian of pion and nucleon fields [12] supplemented with the rela- tivistic $\Delta$-isobar fields [13]. We organize the Lagrangian $\mathcal{L}^{(i)}$, such that superscript $i$ stands for the power of electromagnetic coupling $e$ plus the number of derivatives of pion and photon fields. Writing here only the relevant terms involving the spin-3/2 isospin-3/2 field $\psi^{\mu}$ of the $\Delta$ isobar, we have (with antisymmetric products of $\gamma$ matrices: $\left.\gamma^{\mu \nu}=\frac{1}{2}\left[\gamma^{\mu}, \gamma^{\nu}\right], \gamma^{\mu \nu \alpha}=i \varepsilon^{\mu \nu \alpha \beta} \gamma_{\beta} \gamma_{5}\right)$

$$
\begin{aligned}
\mathcal{L}_{\Delta}^{(1)}= & \bar{\psi}_{\mu}\left(i \gamma^{\mu \nu \alpha} D_{\alpha}-M_{\Delta} \gamma^{\mu \nu}\right) \psi_{\nu} \\
& +\frac{i h_{A}}{2 f_{\pi} M_{\Delta}}\left\{\bar{N} T_{a} \gamma^{\mu \nu \lambda}\left(\partial_{\mu} \psi_{\nu}\right) D_{\lambda} \pi^{a}+\text { H.c. }\right\}, \\
\mathcal{L}_{\Delta}^{(2)}= & \frac{3 i e g_{M}}{2 M\left(M+M_{\Delta}\right)} \bar{N} T_{3} \partial_{\mu} \psi_{\nu} \tilde{F}^{\mu \nu} \\
& -\frac{e h_{A}}{2 f_{\pi} M_{\Delta}} \bar{N} T_{a} \gamma^{\mu \nu \lambda} A_{\mu} \psi_{\nu} \partial_{\lambda} \pi^{a}+\text { H.c., } \\
\mathcal{L}_{\Delta}^{(3)}= & \frac{-3 e}{2 M\left(M+M_{\Delta}\right)} \bar{N} T_{3} \gamma_{5}\left[g_{E}\left(\partial_{\mu} \psi_{\nu}\right) F^{\mu \nu}\right. \\
& \left.+\frac{g_{C}}{M_{\Delta}} \gamma^{\alpha}\left(\partial_{\alpha} \psi_{\nu}-\partial_{\nu} \psi_{\alpha}\right) i \partial_{\mu} F^{\mu \nu}\right]+ \text { H.c., }
\end{aligned}
$$

where $M \simeq 0.939$ and $M_{\Delta} \simeq 1.232 \mathrm{GeV}$ are, respectively, the nucleon and $\Delta$-isobar masses, $N$ and $\pi^{a}(a=1,2,3)$ stand for the nucleon and pion fields, $D_{\mu}$ is the covariant derivative ensuring the electromagnetic gauge-invariance, $F^{\mu \nu}$ and $\tilde{F}^{\mu \nu}$ are the electromagnetic field strength and its dual, $T_{a}$ are the isospin $1 / 2$ to $3 / 2$ transition matrices, and $f_{\pi} \simeq 92.4 \mathrm{MeV}$ is the pion decay constant. $\mathcal{L}_{\Delta}^{(1)}$ contains the Rarita-Schwinger Lagrangian [14] of a free spin-3/2 field formulated such that the number of spin degrees of freedom is constrained to the physical number. The couplings in Eq. (1) are consistent with these constraints because of a spin-3/2 gauge symmetry [15].

We next turn to the power counting for the pion electroproduction amplitude using the " $\delta$-expansion" scheme [13]. In this scheme the excitation energy of the $\Delta$ resonance, namely, $\Delta \equiv M_{\Delta}-M \simeq 0.3 \mathrm{GeV}$, is treated as a light scale, so that for $\Lambda \sim 1 \mathrm{GeV}$ representing the heavy scales in the theory, we can use a small parameter 
$\delta=\Delta / \Lambda$. The other typical light scale of the theory, the pion mass, is counted as two powers of the small parameter: $m_{\pi} / \Lambda \sim \delta^{2}$. The latter rule is the main distinction of this scheme from the previous power countings [16,17] which count $\Delta$ and $m_{\pi}$ at the same order. This difference plays a crucial role in separating the low-energy and resonance regimes, as well as in approaching the chiral limit where $m_{\pi}$ vanishes while $\Delta$ remains finite. Because of the distinction of $m_{\pi}$ and $\Delta$, the counting of a given diagram depends on whether the characteristic momentum $p$ is in the low-energy region $\left(p \sim m_{\pi}\right)$ or in the resonance region $(p \sim \Delta)$. In the resonance region, one distinguishes the one- $\Delta$-reducible $(\mathrm{O} \Delta \mathrm{R})$ graphs [13]; see, e.g., graph (a) in Fig. 1. Such graphs contain $\Delta$ propagators which go as $1 /(p-\Delta)$ and hence for $p \sim \Delta$ they are large and all need to be included. Their resummation amounts to dressing the $\Delta$ propagators so that they behave as $1 /(p-\Delta-\Sigma)$. The self-energy $\Sigma$ begins at order $p^{3}$, and thus a dressed $\mathrm{O} \Delta \mathrm{R}$ propagator counts as $1 / \delta^{3}$.

The pion electroproduction amplitude to next-to-leading order (NLO) in the $\delta$ expansion, in the resonance region, is thus given by the graphs in Figs. 1(a) and 1(b) where the shaded blobs in graph (a) include corrections depicted in Figs. 1(c)-1(f). The hadronic part of graph (a) begins at $\mathcal{O}\left(\delta^{0}\right)$, which here is the leading order. The Born graphs [Fig. 1(b)] contribute at $\mathcal{O}(\delta)$. We note that at NLO there are also vertex corrections of the type (e) and (f) with nucleon propagators in the loop replaced by the $\Delta$ propagators. However, adopting the on-mass shell renormalizations and $Q^{2} \ll \Lambda \Delta$, these graphs start to contribute at next-to-next-to-leading order (NNLO).

We have not shown the $\gamma N \Delta$-vertex correction graph where the photon couples into the $\pi N N$ vertex, because at this order the effect of this graph can be fully absorbed in the graphs in Figs. 1(e) and 1(f) by a field redefinition relating the pseudovector and pseudoscalar $\pi N N$ couplings. Having done that, we compute graphs in Figs. 1(e) and 1(f) using the pseudoscalar coupling.

The self-energy correction [Fig. 1(c)] was computed previously [18]. In that calculation, the experimental value for the $\Delta$-resonance width fixes $h_{A} \simeq 2.85$. To present the results for the vertex corrections, we first consider the general form of the $\gamma N \Delta$ vertex:

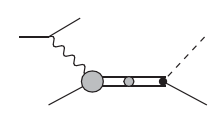

(a)

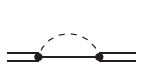

(c)
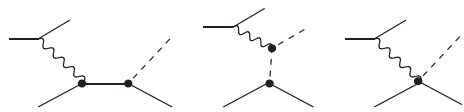

(b)

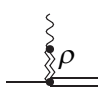

(d)

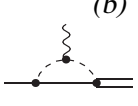

(e)

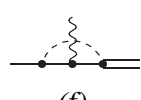

(f)
FIG. 1. Diagrams for the $e N \rightarrow e \pi N$ reaction at NLO in the $\delta$ expansion, considered in this work. Double lines represent the $\Delta$ propagators. The crossed nucleon-exchange graph is not shown in (b) but is included in the calculation.

$$
\begin{aligned}
\bar{u}_{\alpha}\left(p^{\prime}\right) \Gamma_{\gamma N \Delta}^{\alpha \mu} u(p)= & \sqrt{\frac{3}{2}} \frac{M_{\Delta}+M}{M\left[\left(M_{\Delta}+M\right)^{2}+Q^{2}\right]} \bar{u}_{\alpha}\left(p^{\prime}\right) \\
& \times\left\{g_{M}\left(Q^{2}\right) \varepsilon^{\alpha \mu \kappa \lambda} p_{\kappa}^{\prime} q_{\lambda}+g_{E}\left(Q^{2}\right)\right. \\
& \times\left(q^{\alpha} p^{\prime \mu}-q \cdot p^{\prime} g^{\alpha \mu}\right) i \gamma_{5}+g_{C} \\
& \left.\times\left(Q^{2}\right)\left(q^{\alpha} q^{\mu}-q^{2} g^{\alpha \mu}\right) i \gamma_{5}\right\} u(p),
\end{aligned}
$$

where $u_{\alpha}$ is the $\Delta$ vector spinor, $u$ is the nucleon spinor, $q=p^{\prime}-p$ is the photon four-momentum, $Q^{2}=-q^{2}$, and $g_{M}, g_{E}$, and $g_{C}$ are the form factors which at $Q^{2}=0$ are equal to the physical values of corresponding parameters from Lagrangian (1). These form factors relate to the conventional magnetic $\left(G_{M}^{*}\right)$, electric $\left(G_{E}^{*}\right)$ and Coulomb $\left(G_{C}^{*}\right)$ form factors of Jones and Scadron [19] as follows:

$$
\begin{aligned}
G_{M}^{*} & =g_{M}+\frac{M_{\Delta}^{2}}{Q_{+}^{2}}\left(-\beta_{\gamma} g_{E}+\bar{Q}^{2} g_{C}\right), \\
G_{E}^{*} & =\frac{M_{\Delta}^{2}}{Q_{+}^{2}}\left(-\beta_{\gamma} g_{E}+\bar{Q}^{2} g_{C}\right), \\
G_{C}^{*} & =-\frac{2 M_{\Delta}^{2}}{Q_{+}^{2}}\left(g_{E}+\beta_{\gamma} g_{C}\right),
\end{aligned}
$$

where $Q_{ \pm}=\sqrt{\left(\bar{M}_{\Delta} \pm M\right)^{2}+Q^{2}}, \quad \bar{Q}^{2}=Q^{2} / M_{\Delta}^{2}, \quad$ and $\beta_{\gamma}=\frac{1}{2}\left(1-r^{2}-\bar{Q}^{2}\right)$, with $r=M / M_{\Delta}$. The ratios $E 2 / M 1$ and $C 2 / M 1$ at the resonance position can be expressed in terms of these form factors as

$$
R_{E M}=-G_{E}^{*} / G_{M}^{*}, \quad R_{S M}=-\frac{Q_{+} Q_{-}}{4 M_{\Delta}^{2}} G_{C}^{*} / G_{M}^{*} .
$$

The one-loop corrections to the $\gamma N \Delta$ form factors are given by the graphs in Figs. 1(e) and 1(f). For example, the [modified minimal subtraction scheme $(\overline{M S})]$ result for the graph (e) in Fig. 1 can be cast in the form

$$
\begin{aligned}
g_{M}^{(e)}= & -C_{N \Delta} \int_{0}^{1} d y y \int_{0}^{1-y} d x \ln \mathcal{M}^{2}, \\
g_{E}^{(e)}= & +C_{N \Delta} \int_{0}^{1} d y y \int_{0}^{1-y} d x\left\{\ln \mathcal{M}^{2}\right. \\
& \left.-2 x[x r+(1-x-y)(1+r)] \mathcal{M}^{-2}\right\}, \\
g_{C}^{(e)}= & -C_{N \Delta} \int_{0}^{1} d y y(2 y-1) \\
& \times \int_{0}^{1-y} d x[x r+(1-x-y)(1+r)] \mathcal{M}^{-2},
\end{aligned}
$$

where $\quad \mathcal{M}^{2} \equiv(x-\beta)^{2}-\lambda^{2}+2 \beta_{\gamma} x y+\bar{Q}^{2} y(1-y)-$ $i \varepsilon, \quad \mu=m_{\pi} / M_{\Delta}, \quad \beta=\frac{1}{2}\left(1-r^{2}+\mu^{2}\right), \lambda^{2}=\beta^{2}-\mu^{2}$, $C_{N \Delta}=4 g_{A} h_{A} Q_{+}^{2} r^{2} /\left[3(1+r)\left(8 \pi f_{\pi}\right)^{2}\right]$, and $g_{A} \simeq 1.26$. Analogous expressions are obtained for the graph in Fig. 1(f). Alternatively, we have computed these graphs by using the sideways dispersion relations (see, e.g., [20]) and obtained identical results.

The vector-meson diagram [Fig. 1(d)] contributes to NLO for $Q^{2} \sim \Lambda \Delta$. We include it effectively by giving the $g_{M}$ term a dipole $Q^{2}$ dependence (in analogy to how it 
is usually done for the nucleon isovector form factor): $g_{M} \rightarrow g_{M}\left(1+Q^{2} / 0.71 \mathrm{GeV}^{2}\right)^{-2}$. An analogous effect for the $g_{E}$ and $g_{C}$ couplings begins at NNLO and is not included in the present calculation.

We now present the electroproduction observables corresponding to the NLO amplitude of Fig. 1. Denoting the invariant mass of the final $\pi N$ system by $s$, we restrict ourselves to the resonance kinematics: $s=M_{\Delta}^{2}$. The $\gamma^{*} N \rightarrow \pi N$ cross section for unpolarized nucleons are expressed in terms of 5 response functions as

$$
\begin{aligned}
\frac{d \sigma}{d \Omega_{\pi}}= & \frac{d \sigma_{T}}{d \Omega_{\pi}}+\varepsilon \frac{d \sigma_{L}}{d \Omega_{\pi}}+\varepsilon \cos 2 \Phi \frac{d \sigma_{T T}}{d \Omega_{\pi}} \\
& +\sqrt{2 \varepsilon(1+\varepsilon)} \cos \Phi \frac{d \sigma_{L T}}{d \Omega_{\pi}} \\
& +h \sqrt{2 \varepsilon(1-\varepsilon)} \sin \Phi \frac{d \sigma_{L T}^{\prime}}{d \Omega_{\pi}},
\end{aligned}
$$

where $\Theta_{\pi}$ and $\Phi$ are the pion polar and azimuthal c.m. angles, respectively, and $h$ denotes the electron helicity.

In Fig. 2 we show our $\chi$ EFT results for the different cross sections entering Eq. (6). The only free parameters in this calculation are the low-energy constants from Eq. (1), which were chosen to yield the best description of the data as $g_{M}=2.88, g_{E}=-1.04$, and $g_{C}=-2.36$. Within $\chi$ EFT, we can estimate the theoretical uncertainty of the NLO result due to higher-order effects. The NNLO corrections to the amplitudes are expected to be of the order of $\delta^{2}, m_{\pi} / \Lambda$, or $Q^{2} / \Lambda^{2}$. Therefore, the theoretical uncertainty $R_{\text {err }}$ of an observable $R$, which involves a product of two amplitudes, is estimated as (here taking $\Lambda=M$ ):

$$
R_{\mathrm{err}}=2\left|R_{\mathrm{av}}\right| \frac{1}{3}\left(\delta^{2}+\frac{m_{\pi}}{M}+\frac{Q^{2}}{M^{2}}\right),
$$

where $R_{\mathrm{av}}$ is an average value of $R$. In Fig. 2 the average is
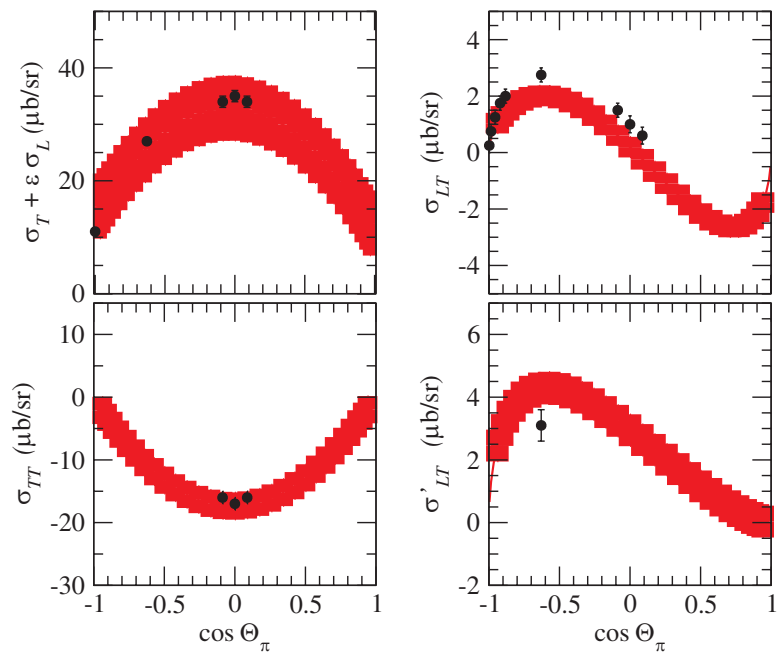

FIG. 2 (color online). $\chi$ EFT NLO results for the $\Theta_{\pi}$ dependence of the $\gamma^{*} p \rightarrow \pi^{0} p$ cross sections at $\sqrt{s}=1.232 \mathrm{GeV}$ and $Q^{2}=0.127 \mathrm{GeV}^{2}$. The theoretical error bands are described in the text. Data points are from BATES experiments $[3,24]$. taken over the range of $\Theta_{\pi}$. One sees that the NLO $\chi$ EFT calculation, within its accuracy, is consistent with the experimental data for these observables.

In Fig. 3 we show the $Q^{2}$ dependence of the ratios $R_{E M}$ and $R_{S M}$. Having fixed the low-energy constants $g_{M}, g_{E}$, and $g_{C}$, the $Q^{2}$ dependence follows as a prediction. The theoretical uncertainty here (shown by the error bands) is estimated according to Eq. (7) with the average $R_{\mathrm{av}}$ taken over the range of $Q^{2}$ from 0 to $0.2 \mathrm{GeV}^{2}$. From the figure, one sees that the NLO calculations are consistent with the experimental data for both of the ratios.

In Fig. 4 we show the $m_{\pi}$ dependence of the $\gamma N \Delta$ transition ratios, with the theoretical uncertainty estimated according to Eq. (7) where $R_{\text {av }}$ is taken over the range of $m_{\pi}^{2}$ from 0 to $0.15 \mathrm{GeV}^{2}$. The study of the $m_{\pi}$ dependence is crucial to connect to the lattice QCD results, which at present can be obtained only for larger pion masses. The recent state-of-the-art lattice calculations of these ratios [11] use a linear, in the quark mass $\left(m_{q} \propto m_{\pi}^{2}\right)$, extrapolation to the physical point, thus assuming that the nonanalytic $m_{q}$ dependencies are negligible. The thus obtained value for $R_{S M}$ at the physical $m_{\pi}$ value displays a large discrepancy with the experimental result, as seen in Fig. 4. However, our calculation demonstrates that the nonanalytic dependencies are not negligible. While at larger values of $m_{\pi}$, where the $\Delta$ is stable, the ratios display a smooth $m_{\pi}$ dependence, at $m_{\pi}=\Delta$ there is an inflection point, and for $m_{\pi} \leq \Delta$ the nonanalytic effects are crucial, as was also observed for the $\Delta$-resonance magnetic moment $[18,21]$. The $m_{\pi}$ dependence obtained in $\chi$ EFT clearly shows that the lattice results for $R_{S M}$ may in fact be consistent with experiment.

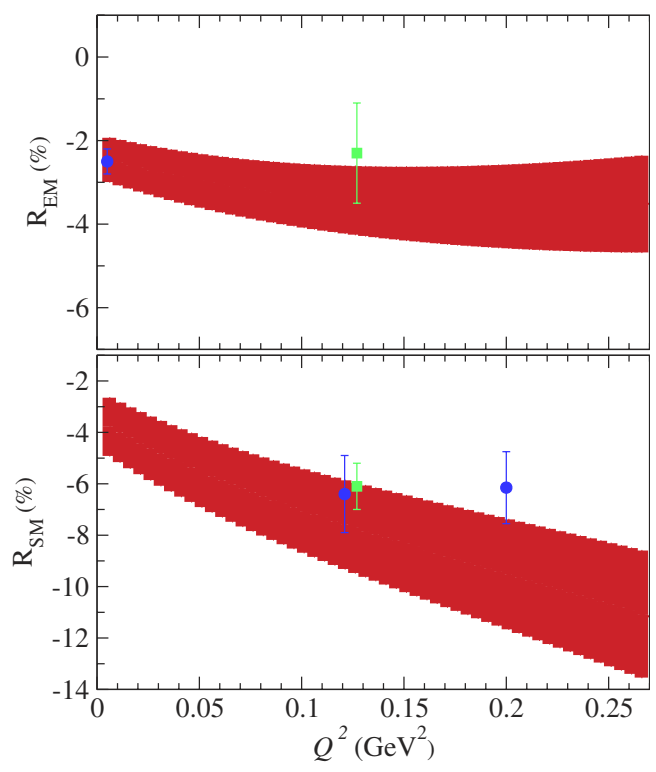

FIG. 3 (color online). $Q^{2}$ dependence of the NLO results for $R_{E M}$ (upper panel) and $R_{S M}$ (lower panel). The blue circles are data points from MAMI for $R_{E M}[1]$ and $R_{S M}[25,26]$. The green squares are data points from BATES [3]. 


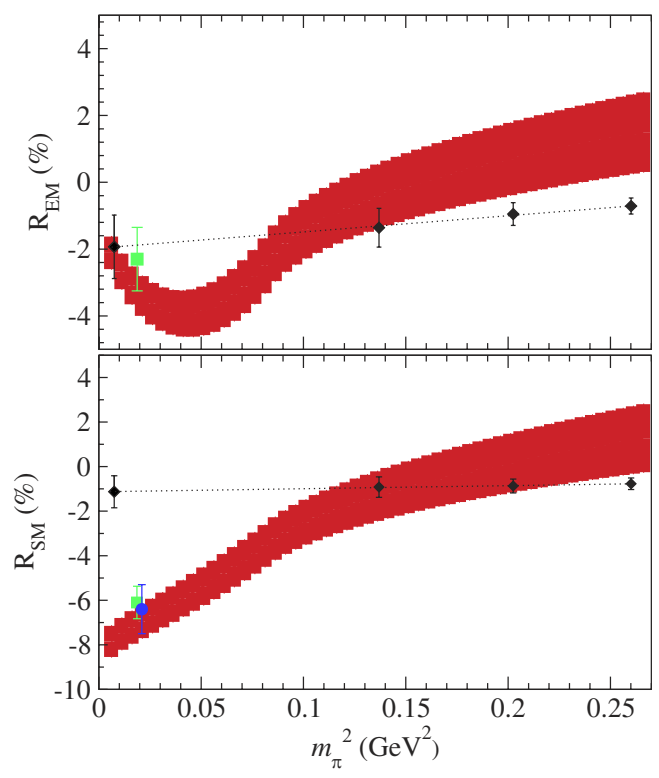

FIG. 4 (color online). $m_{\pi}$ dependence of the NLO results at $Q^{2}=0.1 \mathrm{GeV}^{2}$ for $R_{E M}$ (upper panel) and $R_{S M}$ (lower panel). The blue circle is a data point from MAMI [25]; the green squares are data points from BATES [3]. The solid black diamonds are lattice calculations [11], whereas the dashed lines and open diamonds represent their extrapolation assuming linear dependence in $m_{\pi}^{2}$.

Note that the $m_{\pi}$ dependence of $\Delta$ and $N$ masses was not taken into account in this work. However, we expect this effect to play a minor role in the ratios. In particular, it will not alter the threshold position, $m_{\pi}=\Delta$, because the $\Delta$-nucleon mass splitting stays nearly constant as $m_{\pi}$ increases, as lattice calculations indicate [22].

In conclusion, we have performed a manifestly gaugeand Lorentz-invariant $\chi$ EFT calculation of the $e N \rightarrow e N \pi$ reaction in the $\Delta(1232)$-resonance region. To NLO in the $\delta$ expansion, the only free parameters entering the calculation are the $\gamma N \Delta$ couplings $g_{M}, g_{E}$, and $g_{C}$, characterizing the $M 1, E 2$, and $C 2$ transitions. Our results agree well with recent high-precision data from MAMI and MIT-BATES at low $Q^{2}$. The $\chi$ EFT framework plays a dual role, in that it allows for an extraction of resonance parameters from observables and predicts their $m_{\pi}$ dependence. In this way it may provide a crucial connection of present lattice QCD results obtained at unphysical values of $m_{\pi}$ to the experiment. We have found that the opening of the $\Delta \rightarrow$ $\pi N$ decay channel at $m_{\pi}=M_{\Delta}-M$ induces a pronounced nonanalytic behavior of the $R_{E M}$ and $R_{S M}$ ratios. While the linearly extrapolated lattice QCD results for $R_{S M}$ are in disagreement with experimental data, the $\chi \mathrm{EFT}$ prediction of the nonanalytic dependencies has allowed us to reconcile these results with experiment. As the next-generation lattice calculations of these quantities are on the way [23], the $\chi$ EFT framework presented here will, hopefully, complement these efforts.
This work is supported in part by DOE Grant No. DEFG02-04ER41302 and Contract No. DE-AC05-84ER40150, under which SURA operates Jefferson Lab.

*Electronic address: vlad@jlab.org

${ }^{\dagger}$ Electronic address: marcvdh@jlab.org

[1] R. Beck et al., Phys. Rev. Lett. 78, 606 (1997); Phys. Rev. C 61, 035204 (2000).

[2] G. Blanpied et al., Phys. Rev. Lett. 79, 4337 (1997).

[3] C. Mertz et al., Phys. Rev. Lett. 86, 2963 (2001); N. F. Sparveris et al., ibid. 94, 022003 (2005).

[4] S. Nozawa, B. Blankleider, and T.-S. H. Lee, Nucl. Phys. A513, 459 (1990); T. Sato and T.-S. H. Lee, Phys. Rev. C 54, 2660 (1996); 63, 055201 (2001).

[5] Y. Surya and F. Gross, Phys. Rev. C 53, 2422 (1996).

[6] S. S. Kamalov and S. N. Yang, Phys. Rev. Lett. 83, 4494 (1999); S. Kamalov et al., Phys. Lett. B 522, 27 (2001).

[7] V. Pascalutsa and J.A. Tjon, Phys. Rev. C 70, 035209 (2004); G. Caia et al., ibid. 70, 032201(R) (2004).

[8] C. E. Carlson, Phys. Rev. D 34, 2704 (1986); C. E. Carlson and N.C. Mukhopadhyay, Phys. Rev. Lett. 81, 2646 (1998).

[9] V. V. Frolov et al., Phys. Rev. Lett. 82, 45 (1999); K. Joo et al., ibid. 88, 122001 (2002).

[10] N. Isgur, G. Karl, and R. Koniuk, Phys. Rev. D 25, 2394 (1982); S. Capstick and G. Karl, ibid. 41, 2767 (1990); G. A. Miller, Phys. Rev. C 68, 022201(R) (2003); A. M. Bernstein, Eur. Phys. J. A 17, 349 (2003).

[11] C. Alexandrou et al., Phys. Rev. Lett. 94, 021601 (2005).

[12] J. Gasser, M. E. Sainio, and A. Svarc, Nucl. Phys. B307, 779 (1988); J. Gegelia et al., J. Phys. G 29, 2303 (2003).

[13] V. Pascalutsa and D. R. Phillips, Phys. Rev. C 67, 055202 (2003); 68, 055205 (2003).

[14] W. Rarita and J. S. Schwinger, Phys. Rev. 60, 61 (1941).

[15] V. Pascalutsa, Phys. Rev. D 58, 096002 (1998); Phys. Lett. B 503, 85 (2001); V. Pascalutsa and R. Timmermans, Phys. Rev. C 60, 042201(R) (1999).

[16] E. Jenkins and A. V. Manohar, Phys. Lett. B 255, 558 (1991); 259, 353 (1991).

[17] T. Hemmert, B. R. Holstein, and J. Kambor, Phys. Lett. B 395, 89 (1997); G. Gellas et al., Phys. Rev. D 60, 054022 (1999).

[18] V. Pascalutsa and M. Vanderhaeghen, Phys. Rev. Lett. 94, 102003 (2005).

[19] H. F. Jones and M. D. Scadron, Ann. Phys. (N.Y.) 81, 1 (1973).

[20] B. R. Holstein, V. Pascalutsa, and M. Vanderhaeghen, Phys. Rev. D 72, 094014 (2005).

[21] R. D. Young, D. B. Leinweber, and A. W. Thomas, Nucl. Phys. B, Proc. Suppl. 129, 290 (2004).

[22] C. W. Bernard et al., Phys. Rev. D 64, 054506 (2001).

[23] C. Alexandrou et al., hep-lat/0509140.

[24] C. Kunz et al., Phys. Lett. B 564, 21 (2003).

[25] T. Pospischil et al., Phys. Rev. Lett. 86, 2959 (2001).

[26] D. Elsner et al., nucl-ex/0507014. 